\title{
A stability analysis on forced convection boundary layer stagnation-point slip flow in Darcy-Forchheimer porous medium towards a shrinking sheet
}

\begin{abstract}
The stagnation-point flow over a shrinking sheet in Darcy-Forchheimer porous medium is numerically studied. The governing partial differential equations are transformed into ordinary differential equations using a similarity transformation, and then solved numerically by using shooting technique method with Maple implementation. Dual solutions are observed in a certain range of the shrinking parameter. Regarding on numerical solutions, we prepared stability analysis to identify which solution is stable between non-unique solutions by bvp4c solver in Matlab. Further we obtain numerical results or each solution, which enable us to discuss the features of the respective solutions.
\end{abstract}

Keyword: Stagnation-point flow; Shrinking sheet; Darcy-Forchheimer porous medium; Stability analysis 